Original Article

\title{
Whole blood fatty acid composition at birth: From the maternal compartment to the infant
}

\author{
Carlo Agostoni ${ }^{\mathrm{a}, *}$, Claudio Galli ${ }^{\mathrm{c}}$, Enrica Riva ${ }^{\mathrm{b}}$, Patrizia Risé ${ }^{\mathrm{c}}$, Claudio Colombo ${ }^{\mathrm{c}}$, Marcello Giovannini ${ }^{\mathrm{b}}$, \\ Franca Marangoni ${ }^{\mathrm{c}}$ \\ a Department of Maternal and Pediatric Sciences, University of Milan, Fondazione IRCCS Cà Granda - Ospedale Maggiore Policlinico, Milan, Italy \\ ${ }^{\mathrm{b}}$ Department of Pediatrics, San Paolo Hospital, University of Milan, Milan, Italy \\ ${ }^{\mathrm{c}}$ Department of Pharmacological Sciences, University of Milan, Milan, Italy
}

\section{A R T I C L E I N F O}

Article history:

Received 13 August 2009

Accepted 31 January 2011

\section{Keywords:}

Fatty acids

Long-chain polyunsaturated fatty acids

Pregnancy

Cord blood

Newborn infants

\section{S U M M A R Y}

Background $\mathcal{E}$ aims: The biological role of fatty acids (FA) in the perinatal period is under active investigation. We here describe the application of a simplified microanalytical procedure to compare the FA profile of maternal, umbilical cord and infant whole blood, inclusive of all circulating lipid fractions and cells.

Methods: The FA composition has been analyzed with a micromethod in 16 triplets, including maternal blood, cord blood at delivery and infant blood at day 4 , respectively.

Results: As expected, the FA composition of blood samples withdrawn from the umbilical cord is more similar to the FA composition of blood from 4-day old infants than the FA pattern of maternal blood at delivery. Nevertheless, infant blood FA profile differed from cord for lower long-chain polyunsaturated FA and higher monounsaturated FA.

Conclusions: Our explorative data using whole blood microanalysis confirm the progressive increase of long-chain polyunsaturated FA levels from the mothers towards cord and then infant blood.

(C) 2011 Elsevier Ltd and European Society for Clinical Nutrition and Metabolism. All rights reserved.

\section{Introduction}

The relevance of the essential polyunsaturated essential fatty acids (PUFA), i.e. linoleic acid (LA, 18:2 n-6) and alpha linolenic acid (ALA, 18:3 n-3), and of their long-chain polyunsaturated fatty acid (LC-PUFA) derivatives for the structural and functional development of brain cortex and retina has been supported by a lot of data. ${ }^{1}$

The amounts of arachidonic (AA, 20:4 n-6) and docosahexaenoic (DHA, 22:6n-3) acids supplied to the foetus by the mother throughout pregnancy are strictly dependent on maternal dietary intakes and metabolic processes taking place between the maternal and infant compartments, respectively, at the placenta level. Although the mechanisms at the cellular level are not fully elucidated yet, the knowledge of the inter-relationships between these compartments could be improved by the availability of analytical methods for the assessment of the FA pattern in large population groups. Finally, even if the composition of cord lipids has been repeatedly analysed, ${ }^{2}$ few studies have explored the relationships

\footnotetext{
* Corresponding author. Tel.: +39 02 55032452; fax: +39 0250320226

E-mail address: carlo.agostoni@unimi.it (C. Agostoni).
}

between maternal/cord FA and the FA composition of infant blood lipid classes, for either ethical and practical reasons.

We here describe the application of a simplified microanalytical procedure to compare the FA profile of maternal, umbilical cord and infant whole blood, as representative of all circulating lipid fractions and cells.

\section{Methods}

Sixteen healthy pregnant women aged 34 years (range 28-40), who delivered healthy term infants (38.5 weeks, range 37.5-39.3), with a weight appropriate for gestational age were recruited in our maternity ward. Exclusion criteria included alcohol consumption and smoking habits in order to avoid possible effects on the FA status. All mothers signed written informed consent for the three blood sampling procedures. The study had been previously approved by the Local Committee on Ethics.

Cord venous blood drops were collected immediately after infant delivery and after clamping of the cord. Maternal blood drops were withdrawn through the puncture of a fingertip, performed with an automatic lancing device equipped with lancets. Infant blood samples were collected from a heel prick at the same time of the Guthrie neonatal screening test on day 4 of life. All 
the infants were breast-fed, and didn't receive any supplemental formula.

All the samples were adsorbed on strips of chromatography paper, following an innovative method validated for reproducibility $^{3}$ and comparison with plasma and cell FA profiles, ${ }^{4}$ and already applied to studies in adults ${ }^{5}$ and infants. ${ }^{6,7}$ All the samples were directly subjected to transmethylation for gaschromatographic analysis using DANI GC 1000 equipped with an Omegawax $\mathrm{TM}_{320}$ capillary column ( $30 \mathrm{~m}$, ID $0.32 \mathrm{~mm}$, df $0.25 \mu \mathrm{m}$ ) and FID as detector. FA were recognized by the use of reference standards and values were expressed as FA weight percentages.

The SPSS package 16.0 for Windows (SPSS, Chicago, IL) was used for the statistical analyses. Data (expressed as median, minimum and maximum) were compared with non-parametric tests for 3 related samples (Friedman test) and post-hoc analysis with Dunn's test. Inter-relationships between FA were investigated with the non-parametric Spearman's r.

\section{Results}

Infant characteristics at birth (median and range) were as follows: weight $3.250(2.760-4.010)$ gr, length $50(47-54) \mathrm{cm}$ and head circumference $34(33-36) \mathrm{cm}$. The FA composition of whole blood in the three analysed compartments is reported in Table 1.

As expected, the FA composition of blood samples withdrawn from the umbilical cord is more similar to the FA composition of blood from 4-day old infants than the FA pattern of maternal blood at delivery. Nevertheless, differences between infant and cord blood FA composition are present, with lower LC-PUFA and higher monounsaturated FA. Di-homo-gamma linolenic acid (DHGLA, 20:3 n-6), AA and DHA levels in blood from cord are higher, but in the same order of magnitude than in infant's samples, and are around 2-folds higher than in maternal blood. Conversely, the levels of LA and ALA are 3- to 4-folds lower in cord and infant blood, compared to mothers.

Considering the main product/precursor ratios, representative of the different metabolic steps involved in LC-PUFA biosynthesis of the two metabolic series, regulated by desaturase and elongase reactions, the ratios of the two main products (AA and DHA) with their 18 carbon precursors (LA and ALA, respectively) are similar in cord and infant blood. Nevertheless, considering the ratios of AA and DHA with the major intermediates (DHGLA and eicosapentaeoic acid, EPA, 20:5 n-3, respectively), the AA/DHGLA ratio (on the n-6 pathway) is higher in infant blood while the DHA/EPA ratio (on the $n-3$ pathway) is higher in cord blood.

Mutual inter-relationships have been also investigated for LA, ALA, AA, EPA and DHA values for maternal vs cord, maternal vs neonatal, and cord vs neonatal values, respectively. As concerns maternal vs cord values significant relationships have been found for LA $(r=0.575, P=0.020)$ and EPA $(r=0.673, P=0.004)$, while as far as cord $v s$ neonatal percentage concentrations, a significant correlation has been found only for LA $(r=0.526, P=0.036)$. No other relations between the levels of the same fatty acid and the three compartments have been found.

\section{Discussion}

To our knowledge, this is the first study investigating the FA composition of whole blood in triplets of mothers, cord and infants. Other previous studies have considered the relationship between the maternal compartments and the infants by analysing plasma total lipids or phospholipids or red blood cells. However whole blood lipids are representative of the FA composition of all the circulating lipid classes, lipoproteins and cells. Our observations confirm previous observations with different methodology on the progressively higher levels of LC-PUFA from mothers towards cord and infants, respectively. ${ }^{8}$ In this context, the infant FA profile only marginally reflects the maternal FA status, partly depending on the maternal release and partly upon the metabolism in the placenta or the infant. The biomechanisms at the basis of these differences are actually under active investigation. In spite of the documented LCPUFA biosynthesis in both preterm and term newborns, ${ }^{9}$ the two major LC-PUFA (AA and DHA) in whole blood are already decreased at four days of life compared to cord levels Consumption and/or utilization rates higher than synthetic capacities of infants might explain these observations. Of interest, maternal n-3 LC-PUFA levels (including both EPA and DHA) are related to the n-3 LC-PUFA levels of cord but not of infants.

We conclude that, consistent with the explorative purposes of our study, the micro invasive and microanalytical method for the whole blood FA analysis seems to be a useful tool to obtain further information concerning fatty acids from larger populations and

Table 1

Percentage levels of the main individual FA, classes and product/precursor ratios ( $n=16$; medians, min-max) in the different blood samples.

\begin{tabular}{|c|c|c|c|c|c|c|c|c|c|}
\hline \multirow[t]{2}{*}{ FA } & \multicolumn{3}{|c|}{ Maternal } & \multicolumn{3}{|l|}{ Cord } & \multicolumn{3}{|l|}{ Infant } \\
\hline & Median & Min & Max & Median & Min & Max & Median & Min & Max \\
\hline $18: 0$ & 7.47 & 6.40 & 10.4 & 16.9 & 15.5 & 18.0 & 15.0 & 12.4 & 16.4 \\
\hline $18: 1 \mathrm{n}-9$ & 24.1 & 18.4 & 29.6 & 13.0 & 11.7 & 15.3 & 16.9 & 13.6 & 21.7 \\
\hline $18: 2 n-6$ & 20.4 & 15.4 & 29.7 & $5.61^{\mathrm{ns}}$ & 4.14 & 7.17 & $4.88^{\mathrm{ns}}$ & 3.08 & 9.16 \\
\hline $20: 3 n-6$ & $1.36^{\mathrm{ns}}$ & 1.08 & 2.33 & 2.51 & 1.73 & 3.19 & $1.50^{\mathrm{ns}}$ & 1.32 & 2.03 \\
\hline $20: 4 n-6$ & 6.36 & 3.64 & 8.16 & 14.6 & 12.9 & 15.9 & 13.1 & 9.81 & 16.0 \\
\hline $18: 3 n-3$ & 0.20 & 0.15 & 0.71 & $0.08^{\mathrm{ns}}$ & 0.04 & 0.33 & $0.08^{\mathrm{ns}}$ & 0.03 & 0.21 \\
\hline $20: 5 n-3$ & 0.15 & 0.07 & 0.29 & 0.08 & 0.03 & 0.14 & 0.10 & 0.05 & 0.23 \\
\hline $22: 6 n-3$ & 1.70 & 0.69 & 2.68 & 3.88 & 1.90 & 4.93 & 3.28 & 1.63 & 3.95 \\
\hline Saturated & 38.6 & 35.0 & 42.5 & $50.6^{\mathrm{ns}}$ & 46.8 & 52.9 & $51.1^{\mathrm{ns}}$ & 47.5 & 54.4 \\
\hline Monounsaturated & 28.8 & 22.5 & 34.7 & 18.2 & 16.7 & 21.9 & 22.7 & 18.6 & 28.0 \\
\hline Polyunsaturated & $32.3^{\text {ns }}$ & 26.0 & 42.0 & $31.0^{\mathrm{ns}}$ & 26.5 & 33.3 & 26.4 & 21.5 & 30.0 \\
\hline Total n-6 & 29.1 & 23.6 & 38.3 & 26.6 & 22.8 & 28.4 & 22.2 & 18.3 & 25.6 \\
\hline Total n-3 & 2.36 & 1.29 & 3.75 & $4.37^{\mathrm{ns}}$ & 2.39 & 5.48 & $3.74^{\mathrm{ns}}$ & 2.13 & 4.46 \\
\hline DHGLA/LA & 0.07 & 0.04 & 0.13 & 0.46 & 0.29 & 0.72 & 0.28 & 0.18 & 0.66 \\
\hline AA/DHGLA & 4.54 & 2.79 & 6.17 & 5.89 & 4.38 & 7.67 & 8.53 & 5.81 & 10.7 \\
\hline $\mathrm{AA} / \mathrm{LA}$ & 0.29 & 0.18 & 0.42 & $2.61^{\mathrm{ns}}$ & 1.90 & 3.74 & $2.72^{\mathrm{ns}}$ & 1.17 & 4.10 \\
\hline EPA/ALA & 0.61 & 0.29 & 1.13 & $1.04^{\mathrm{ns}}$ & 0.30 & 2.28 & $1.44^{\mathrm{ns}}$ & 0.53 & 2.39 \\
\hline DHA/ALA & 7.86 & 2.86 & 12.0 & $45.0^{\mathrm{ns}}$ & 10.9 & 89.7 & $47.3^{\mathrm{ns}}$ & 11.7 & 114.8 \\
\hline DHA/EPA & 12.4 & 4.93 & 0.13 & 41.6 & 28.0 & 133.3 & 32.5 & 10.6 & 66.8 \\
\hline
\end{tabular}

Friedman test (3 related samples, non-parametric). ${ }^{\text {ns }}$ indicates the absence of statistically significant between-group differences at post-hoc analysis (Dunn's test). 
elucidate the mechanisms regulating the transfer/distribution of PUFA within the maternal-infant dyad.

\section{Conflict of interest}

The authors have no conflict of interest to declare.

\section{Acknowledgements}

Authors' contribution: Carlo Agostoni and Franca Marangoni analysed the data and wrote the manuscript. Franca Marangoni, Patrizia Risé and Claudio Colombo analysed the blood samples. Claudio Galli, Enrica Riva and Marcello Giovannini proposed the study design, organized the study with the collection of blood samples, and finally revised the manuscript.

\section{References}

1. Uauy R, Hoffman DR, Peirano P, Birch DG, Birch EE. Essential fatty acids in visual and brain development. Lipids 2001;36:885-95.
2. Minda H, Larque E, Koletzko B, Decsi T. Systematic review of fatty acid composition of plasma phospholipids of venous cord blood in full-term infants. Eur J Nutr 2002;41:125-31.

3. Marangoni F, Colombo C, Galli C. A method for the direct evaluation of the fatty acid status in a drop of blood from a fingertip in humans: applicability to nutritional and epidemiological studies. Anal Biochem 2004;326:267-72.

4. Risé P, Eligini S, Ghezzi S, Colli S, Galli C. Fatty acid composition of plasma, blood cells and whole blood: relevance for the assessment of the fatty acid status in humans. Prostaglandins Leukot Essent Fatty Acids 2007;76:363-9.

5. Marangoni F, Colombo C, Martiello A, Negri E, Galli C. The fatty acid profiles in a drop of blood from a fingertip correlate with physiological, dietary and lifestyle parameters in volunteers. Prostaglandins Leukot Essent Fatty Acids 2007;76:87-92.

6. Agostoni C, Marangoni F, Stival G, Gatelli I, Pinto F, Risé P, et al. Whole blood fatty acid composition differs in term versus mildly preterm infants: small versus matched appropriate for gestational age. Pediatr Res 2008;64:298-302.

7. Agostoni C, Riva E, Giovannini M, Pinto F, Colombo C, Risé P, et al. Maternal smoking habits are associated with differences in infants' long-chain polyunsaturated fatty acids in whole blood: a case-control study. Arch Dis Child 2008;93:414-8.

8. Ghebremeskel K, Crawford MA, Lowy C, Min Y, Thomas B, Golfetto D, et al. Arachidonic and docosahexaenoic acids are strongly associated in maternal and neonatal blood. Eur J Clin Nutr 2000;54:50-6.

9. Uauy R, Mena P, Wegher B, Nieto S, Salem N. Long chain polyunsaturated fatty acid formation in neonates: effect of gestational age and intrauterine growth. Pediatr Res 2000;47:127-35. 